\title{
STABILITY OF A LINEAR INTEGRO-DIFFERENTIAL EQUATION WITH PERIODIC COEFFICIENTS
}

\author{
BY \\ ALEKSEY D. DROZDOV AND MICHAEL I. GIL \\ Institute for Industrial Mathematics, Ben-Gurion University of the Negev, Beer-Sheva, Israel
}

\begin{abstract}
Stability of a linear integro-differential equation with periodic coefficients is studied. Such an equation arises in the dynamics of thin-walled viscoelastic elements of structures under periodic compressive loading. The equation under consideration has a specific peculiarity which makes its analysis difficult: in the absence of the integral term it is only stable, but not asymptotically stable. Therefore, in order to derive stability conditions we have to introduce some specific restrictions on the behavior of the kernel of the integral operator. These restrictions are taken from the analysis of the relaxation measures for a linear viscoelastic material.

We suggest an approach to the study of the stability based on the direct Lyapunov method and construct new stability functionals. Employing these techniques we derive some new sufficient stability conditions which are close enough to the necessary ones. In particular, when the integral term vanishes, our stability conditions pass into the well-known stability criterion for a linear differential equation with periodic coefficients. In the general case, the proposed stability conditions have the following mechanical meaning: a viscoelastic structure under periodic excitations is asymptotically stable if the corresponding elastic structure is stable and the material viscosity is sufficiently large.

As an example, the stability problem is considered for a linear viscoelastic beam compressed by periodic-in-time forces. Explicit limitations on the material parameters are obtained which guarantee the beam stability, and the dependence of the critical relaxation rate on the material viscosity is analysed numerically for different frequencies of the periodic compressive load.
\end{abstract}

1. Introduction. In the past two decades a number of publications were devoted to the study of stability of Volterra integro-differential equations; see, e.g., Burton (1983), Corduneanu and Lakshmikantham (1980), Gripenberg et al. (1990), Hale (1977), Kolmanovskii and Nosov (1986) and the bibliography therein.

One of the main applications of this theory is the stability of viscoelastic bodies and thin-walled elements of structures; see, e.g., Bloom (1984), Coleman and Dill (1968), Dafermos (1970), Dafermos and Nohel (1979), Drozdov et al. (1991), Fabrizio and Lazzari (1991), Fabrizio and Morro (1992), MacCamy (1977), Renardy et al. (1987).

Received July 20, 1993.

1991 Mathematics Subject Classification. Primary 34D20, 34K20.

(C)1996 Brown University 
To our knowledge, in this sphere of applications, the stability conditions have been studied in detail for integro-differential equations with constant coefficients, but up to now there is no general theory in the case of variable, especially periodic, coefficients.

Stability of ordinary differential equations with periodic coefficients was discussed in detail by Erugin (1966), Malkin (1956), Mclachlan (1964), and Yakubovich and Starzhinskii (1975). It was shown that the periodic-in-time loads acting on a system can induce parametric oscillations with unbounded growth of small initial perturbations. This analysis was based on the Floquet representation for the fundamental matrix of a linear differential equation with periodic coefficients. A generalization of the Floquet theory for functional-differential equations was proposed by Stokes (1962).

Parametric instabilities in elastic systems were studied, e.g., by Bolotin (1964), EvanIwanowski (1976), and Herrmann (1967).

Some results in the stability theory for viscoelastic thin-walled elements of structures were obtained by Belen'kaya and Judovich (1978), Cederbaum and Mond (1992), Eshmatov and Kurbanov (1975), Matyash (1967), Stevens (1966). It is worth noting that the stability conditions were obtained in these works either by using simplified constitutive equations reducing the integro-differential equation to the differential one, or by applying approximate methods (averaging techniques, multiple scales analysis, etc.).

The first attempt to derive sufficient stability conditions without any additional simplifications was made by Drozdov (1993) utilizing the direct Lyapunov method. In this paper we develop this method and derive some sufficient stability conditions close enough to the necessary ones.

The exposition will be as follows. In Sec. 2 we formulate the stability problem and some basic assumptions about the behavior of the kernel of the integral operator. In Sec. 3 we construct the stability functionals and derive stability conditions. In Sec. 4 we discuss the conditions obtained and compare them with the stability conditions for the corresponding differential equation. In Sec. 5 we consider the stability problem for a simply supported viscoelastic beam under the action of a periodic compressive load and analyse the influence of the periodic force on the critical rate of relaxation of the material.

2. Formulation of the problem and basic assumptions. Let us consider the following integro-differential equation:

$$
\ddot{u}(t)+a(t) u(t)+b \int_{0}^{t} \dot{Q}(t-s) u(s) d s=0
$$

Here $u(t):[0, \infty) \rightarrow[0, \infty)$ is an unknown function, $a(t)$ is a positive, continuously differentiable, periodic function with the period $T, b$ is a positive constant, $Q(t)$ is the integral kernel. Denote by $a_{0}=T^{-1} \int_{0}^{T} a(t) d t$ the mean value of the function $a(t)$. We suppose that $a_{0}>0$.

The function $Q(t)$ is assumed to be twice continuously differentiable and to satisfy the following conditions: 
(i) for any $t \geq 0$ :

$$
\begin{aligned}
-1<Q(\infty)<Q(t)<Q(0) & =0 \\
\dot{Q}(t) \leq \dot{Q}(\infty) & =0 \\
\ddot{Q}(t) \geq \ddot{Q}(\infty) & =0
\end{aligned}
$$

(ii) there exist positive constants $T_{1}$ and $T_{2}$ such that for any $T \geq 0$ :

$$
T_{2}^{-1}|\dot{Q}(t)| \leq \ddot{Q}(t) \leq T_{1}^{-1}|\dot{Q}(t)|
$$

Equation (1) describes the mechanical behavior of viscoelastic elements of structures under dynamic loading; cf. Drozdov et al. (1991). In these applications, the function $u(t)$ determines the characteristic deflection of a structure, and the function $Q(t)$ determines the relaxation kernel of material. The mechanical meaning of the conditions (i) and (ii) was discussed in Drozdov and Kolmanovskii (1992).

We will employ below the following.

Lemma. Suppose that the conditions (i) and (ii) are fulfilled. Then for any $t \geq 0$ :

$$
-Q(\infty) \exp \left(-t / T_{1}\right) \leq Q(t)-Q(\infty) \leq-Q(\infty) \exp \left(-t / T_{2}\right)
$$

Proof. Taking into account (i) we can rewrite (ii) as follows:

$$
-T_{2}^{-1} \dot{Q}(t) \leq \ddot{Q}(t) \leq-T_{1}^{-1} \dot{Q}(t)
$$

Integrating this inequality from $t$ to infinity and using (i) we find

$$
T_{2}^{-1}[Q(t)-Q(\infty)] \leq-\dot{Q}(t) \leq T_{1}^{-1}[Q(t)-Q(\infty)]
$$

From (3) it follows that

$$
-\frac{d t}{T_{1}} \leq \frac{d Q(t)}{Q(t)-Q(\infty)} \leq-\frac{d t}{T_{2}} .
$$

Integration of (4) from 0 to $t$ with the use of (i) yields (2).

Let us consider the differential equation

$$
\ddot{u}+a(t) u=0
$$

which corresponds to the case when the integral term in (1) vanishes. In the new variables $u_{1}=u(t), u_{2}=\dot{u}(t)$ Eq. (5) can be written in the matrix form:

$$
\dot{U}=A(t) U
$$

where

$$
U=\left[\begin{array}{l}
u_{1} \\
u_{2}
\end{array}\right], \quad A(t)=\left[\begin{array}{cc}
0 & 1 \\
-a(t) & 0
\end{array}\right]
$$


Introduce the vector-function $V(t)$ which satisfies the adjoint differential equation

$$
\dot{V}=-A^{*}(t) V
$$

where the superscript asterisk denotes transpose. Let

$$
\Phi(t)=\left[\begin{array}{ll}
\Phi_{11}(t) & \Phi_{21}(t) \\
\Phi_{12}(t) & \Phi_{22}(t)
\end{array}\right]
$$

be the fundamental matrix for Eq. (7), i.e., the solution of (7) with the initial condition $V(0)=I$, where $I$ is the unit matrix. Here we employ nonstandard notation for elements of the matrix $\Phi(t)$ in order to emphasize that the functions $\Phi_{i j}(t)$ staying in any column of this matrix satisfy independent scalar equations:

$$
\begin{array}{ll}
\dot{\Phi}_{11}=a(t) \Phi_{12}, & \dot{\Phi}_{12}=-\Phi_{11}, \\
\dot{\Phi}_{21}=a(t) \Phi_{22}, & \dot{\Phi}_{22}=-\Phi_{21} .
\end{array}
$$

For a constant coefficient $a=a_{0}$, the matrix $\Phi(t)$ can be represented as follows:

$$
\Phi(t)=\left[\begin{array}{cc}
\cos \sqrt{a_{0}} t & \sqrt{a_{0}} \sin \sqrt{a_{0}} t \\
-\frac{1}{\sqrt{a_{0}}} \sin \sqrt{a_{0}} t & \cos \sqrt{a_{0}} t
\end{array}\right] .
$$

Similarly, let

$$
\Psi(t)=\left[\begin{array}{ll}
\Psi_{11}(t) & \Psi_{21}(t) \\
\Psi_{12}(t) & \Psi_{22}(t)
\end{array}\right]
$$

be the fundamental matrix for Eq. (6). One can show that

$$
\begin{array}{ll}
\Psi_{11}(t)=\Phi_{22}(t), & \Psi_{12}(t)=-\Phi_{21}(t), \\
\Psi_{21}(t)=-\Phi_{12}(t), & \Psi_{22}(t)=\Phi_{11}(t) .
\end{array}
$$

From the Liouville theorem it follows that for any $t \geq 0$ :

$$
\operatorname{det} \Psi(t)=\operatorname{det} \Phi(t)=1
$$

Using this fact we can write the characteristic equation for the matrix $\Psi(T)$ as follows:

$$
\lambda^{2}-I_{1}(\Psi(T)) \lambda+1=0
$$

where $I_{1}$ is the first invariant (trace) of the matrix.

It is well known, see, e.g., Yakubovich and Starzhinskii (1975), that the zero solution of (5) is stable if and only if all eigenvalues of the matrix $\Psi(T)$ are single and lie on the unit circle. Hence, according to (12), the zero solution of (5) is stable iff

$$
\left|I_{1}(\Psi(T))\right|<2 .
$$

Our goal is to generalize this result to the integro-differential equation (1). Namely, we will derive conditions of asymptotic stability for the zero solution of Eq. (1), which turn into the criterion (13) when the integral term in (1) tends to zero. 
3. Stability conditions. In this section we derive some sufficient conditions of asymptotic stability of the zero solution of Eq. (1). In order to formulate these conditions let us introduce the following notation. Denote by $f_{i}(t)$ the functions:

$$
\begin{aligned}
& f_{1}(t)=a_{0} \Phi_{11}(t) \Phi_{12}(t)+\Phi_{21}(t) \Phi_{22}(t) \\
& f_{2}(t)=a_{0} \Phi_{12}^{2}(t)+\Phi_{22}^{2}(t) \\
& f_{3}(t)=a_{0} \Phi_{11}^{2}(t)+\Phi_{21}^{2}(t)
\end{aligned}
$$

From these formulae and (8) it follows that the functions $f_{i}(t)$ satisfy the linear differential equations

$$
\dot{f}_{1}=a(t) f_{2}-f_{3}, \quad \dot{f}_{2}=-2 f_{1}(t), \quad \dot{f}_{3}=2 a(t) f_{1}
$$

with the initial conditions $f_{1}(0)=0, f_{2}(0)=1, f_{3}(0)=a_{0}$. It can be shown that for a constant $a=a_{0}$ :

$$
f_{1}(t)=0, \quad f_{2}(t)=1, \quad f_{3}(t)=a_{0} .
$$

Let $\bar{a}(t)=a(t)+b Q(\infty)$. We assume that

$$
\kappa=\min _{t \geq 0} \bar{a}(t)>0
$$

Introduce the functions

$$
F(t)=\frac{\max \{\dot{a}(t), 0\}}{\bar{a}(t) b}, \quad F_{1}(t)=\frac{f_{1}(t)}{\bar{a}(t)}, \quad F_{2}(t)=\frac{f_{2}(t)}{\bar{a}(t)} .
$$

Denote their maximal and minimal values by $Y, Y_{1}$, and $Y_{2}$ :

$$
Y=\sup _{t \geq 0} F(t), \quad Y_{1}=\sup _{t \geq 0}\left|F_{1}(t)\right|, \quad Y_{2}=\inf _{t \geq 0} F_{2}(t)
$$

Let $H(t)=\int_{0}^{t}[Q(s)-Q(\infty)] d s$. From (2) it follows that the function $H(t)$ is bounded. ThEOREM. Suppose that the conditions (i), (ii) are fulfilled, the inequality (16) holds,

$$
Y_{1}<\min \left\{\frac{3}{4}, \frac{H(\infty)}{H(\infty)+T_{1}|Q(\infty)|}\right\} \alpha^{-1} Y_{2}
$$

and

$$
Y<\frac{H(\infty) Y_{2}-\alpha\left[H(\infty)+T_{1}|Q(\infty)|\right] Y_{1}}{Y_{2}-\alpha Y_{1}}
$$

where $\alpha=3 T_{1}^{-1} T_{2}^{2}$. Then the zero solution of Eq. (1) is asymptotically stable.

Proof. In order to prove this theorem we construct the Lyapunov functionals $L_{1}(t)$, $\ldots, L_{6}(t)$ such that the function $L_{6}(t)$ is positive and nonincreasing in time.

Let us introduce the new variables

$$
z_{1}=u_{1} \Phi_{11}(t)+u_{2} \Phi_{12}(t), \quad z_{2}=u_{1} \Phi_{21}(t)+u_{2} \Phi_{22}(t)
$$


Differentiating these expressions and using (1) and (8) we obtain

$$
\dot{z}_{1}=-b \Phi_{12}(t) \int_{0}^{t} \dot{Q}(t-s) u_{1}(s) d s, \quad \dot{z}_{2}=-b \Phi_{22}(t) \int_{0}^{t} \dot{Q}(t-s) u_{1}(s) d s .
$$

Let us consider the function

$$
L_{1}(t)=\frac{1}{2}\left[a_{0} z_{1}^{2}(t)+z_{2}^{2}(t)\right]
$$

For a constant $a=a_{0}$, employing (9) we find that $L_{1}=\frac{1}{2}\left[a_{0} u^{2}(t)+\dot{u}^{2}(t)\right]$, i.e., it equals the total energy of the conservative system without the integral term. Differentiation of (20) with the use of (19) yields

$$
\dot{L}_{1}=-b\left[f_{1}(t) u(t)+f_{2}(t) \dot{u}(t)\right] \int_{0}^{t} \dot{Q}(t-s) u(s) d s .
$$

In order to transform the second term in (21) we introduce the functional

$$
L_{2}(t)=\int_{0}^{t} \dot{Q}(t-s)(u(t)-u(s))^{2} d s-Q(t) u^{2}(t)
$$

Differentiating (22) and using (1) and the conditions (i) we have

$$
\dot{L}_{2}=\int_{0}^{t} \ddot{Q}(t-s)(u(t)-u(s))^{2} d s-2 \dot{u}(t) \int_{0}^{t} \dot{Q}(t-s) u(s) d s-\dot{Q}(t) u^{2}(t) .
$$

It follows from (15), (21), and (23) that the derivative of the functional

$$
L_{3}=2 L_{1}(t)-b f_{2}(t) L_{2}(t)
$$

can be represented as follows:

$$
\begin{aligned}
\dot{L}_{3}(t)=2 b f_{1}(t)\left[L_{2}(t)-u(t) \int_{0}^{t} \dot{Q}(t-s) u(s) d s\right] & \\
& -b f_{2}(t)\left[\int_{0}^{t} \ddot{Q}(t-s)(u(t)-u(s))^{2} d s-\dot{Q}(t) u^{2}(t)\right] .
\end{aligned}
$$

Since

$$
-u(t) u(s)=\frac{1}{2}\left[(u(t)-u(s))^{2}-u^{2}(t)-u^{2}(s)\right],
$$

the latter formula has the form

$$
\begin{aligned}
\dot{L}_{3}(t)=b f_{1}(t)\left[3 L_{2}(t)-\int_{0}^{t}\right. & \left.\dot{Q}(t-s) u^{2}(s) d s\right] \\
& -b f_{2}(t)\left[\int_{0}^{t} \ddot{Q}(t-s)(u(t)-u(s))^{2} d s-\dot{Q}(t) u^{2}(t)\right] .
\end{aligned}
$$


Let us consider now the functional

$$
L_{4}(t)=\frac{1}{2}\left\{\dot{u}(t)+b \int_{0}^{t}[Q(t-s)-Q(\infty)] u(s) d s\right\}^{2}
$$

Differentiating (27) and using (1) we obtain

$$
\begin{aligned}
\dot{L}_{4}(t) & =L_{4}(t)\left\{\ddot{u}(t)-b Q(\infty) u(t)+b \int_{0}^{t} \dot{Q}(t-s) u(s) d s\right\} \\
& =-\bar{a}(t) u(t) L_{4}(t) .
\end{aligned}
$$

This relation together with (27) implies that the derivative of the functional

$$
L_{5}(t)=L_{4}(t)+\frac{1}{2} \bar{a}(t) u^{2}(t)
$$

can be calculated as follows:

$$
\dot{L}_{5}(t)=\frac{1}{2} \dot{a}(t) u^{2}(t)-\bar{a}(t) b u(t) \int_{0}^{t}[Q(t-s)-Q(\infty)] u(s) d s .
$$

We transform this equation using (25) and finally obtain

$$
\begin{aligned}
2 \dot{L}_{5}(t)=\dot{a}(t) u^{2}(t)-\bar{a}(t) b\left\{H(t) u^{2}(t)+\right. & \int_{0}^{t}[Q(t-s)-Q(\infty)] u^{2}(s) d s \\
& \left.-\int_{0}^{t}[Q(t-s)-Q(\infty)](u(t)-u(s))^{2} d s\right\} .
\end{aligned}
$$

Let us introduce the functional

$$
L_{6}(t)=L_{3}(t)+2 \beta L_{5}(t)
$$

where $\beta$ is a positive constant which will be determined below. It follows from (26) and (29) that

$$
\begin{aligned}
\dot{L}_{6}(t)=-\bar{a}(t) b\left[G(t) u^{2}(t)+\int_{0}^{t} G_{1}(t, t-s) u^{2}(s) d s\right. & \\
& \left.+\int_{0}^{t} G_{2}(t, t-s)(u(t)-u(s))^{2} d s\right]
\end{aligned}
$$

where

$$
\begin{aligned}
G(t) & =\beta\left[H(t)-\frac{\dot{a}(t)}{\bar{a}(t) b}\right]+3 F_{1}(t) Q(t)-F_{2}(t) \dot{Q}(t), \\
G_{1}(t, \tau) & =\beta[Q(\tau)-Q(\infty)]-F_{1}(t) \dot{Q}(\tau), \\
G_{2}(t, \tau) & =F_{2}(t) \ddot{Q}(\tau)-\beta[Q(\tau)-Q(\infty)]+3 F_{1}(t) \dot{Q}(\tau) .
\end{aligned}
$$


The condition (ii) and (3) imply

$$
T_{2}^{-1}[Q(t)-Q(\infty)] \leq \ddot{Q}(t) \leq T_{1}^{-1}[Q(t)-Q(\infty)]
$$

We estimate the functions $G_{1}$ and $G_{2}$ using this inequality and (3) as follows:

$$
\begin{aligned}
& G_{1}(t, \tau) \geq\left[\beta-T_{1}^{-1}\left|F_{1}(t)\right|\right][Q(\tau)-Q(\infty)] \\
& G_{2}(t, \tau) \geq\left[T_{2}^{-2} F_{2}(t)-3 T_{1}^{-1}\left|F_{1}(t)\right|-\beta\right][Q(\tau)-Q(\infty)] .
\end{aligned}
$$

From these inequalities it follows that the functions $G_{1}(t, \tau)$ and $G_{2}(t, \tau)$ are nonnegative if

$$
Y_{1} \leq \beta T_{1} \leq 3 \alpha^{-1}\left(Y_{2}-\alpha Y_{1}\right)
$$

It follows from (17) that (32) is true and we can put $\beta=3\left(\alpha T_{1}\right)^{-1}\left(Y_{2}-\alpha Y_{1}\right)$. In this case, Eqs. (16) and (31) imply

$$
\dot{L}_{6}(t) \leq-\bar{a}(t) b G(t) u^{2}(t)
$$

Substituting the expressions (24), (28) into (30) and using (20), (22), and (27) we get

$$
\begin{aligned}
L_{6}(t)=a_{0} z_{1}^{2}(t)+ & z_{2}^{2}(t) \\
& -b f_{2}(t)\left[\int_{0}^{t} \dot{Q}(t-s)(u(t)-u(s))^{2} d s-Q(t) u^{2}(t)\right] \\
& +\beta\left\{\left[\dot{u}(t)+b \int_{0}^{t}(Q(t-s)-Q(\infty)) u(s) d s\right]^{2}+\bar{a}(t) u^{2}(t)\right\} .
\end{aligned}
$$

It follows from this formula, (16), and the conditions (i) that

$$
L_{6}(t) \geq \beta \kappa u^{2}(t) \quad(t \geq 0) .
$$

Putting $t=0$ in (34) and utilizing the initial conditions for the matrix function $\Phi(t)$ we find

$$
L_{6}(0)=\left[a_{0}+\beta \bar{a}(0)\right] u^{2}(0)+(1+\beta) \dot{u}^{2}(0) .
$$

Integrating (33) from 0 to $t$ and using (35), (36) we obtain

$$
\beta \kappa u^{2}(t) \leq\left[a_{0}+\beta \bar{a}(0)\right] u^{2}(0)+(1+\beta) \dot{u}^{2}(0)+b \Gamma(t) \int_{0}^{t} u^{2}(s) d s,
$$

where $\Gamma(t)=\max _{0 \leq s \leq t} \bar{a}(s)|G(s)|$. This formula together with the Gronwall inequality implies

$$
\begin{aligned}
u^{2}(t) \leq \frac{1}{\beta \kappa}\left[\left(a_{0}+\beta \bar{a}(0)\right) u^{2}(0)+(1\right. & \left.+\beta) \dot{u}^{2}(0)\right] \\
\times & {\left[1+\frac{b}{\beta \kappa} \Gamma(t) \int_{0}^{t} \exp \left(\frac{b}{\beta \kappa} \int_{s}^{t} \Gamma(\tau) d \tau\right) d s\right] . }
\end{aligned}
$$


Substitution of (37) into (33) yields

$$
\begin{aligned}
\dot{L}_{6}(t) \leq \frac{b}{\beta \kappa}\left[\left(a_{0}+\beta \bar{a}(0)\right) u^{2}(0)+(1+\right. & \left.\beta) \dot{u}^{2}(0)\right] \\
& \times \Gamma(t)\left[1+\frac{b}{\beta \kappa} \Gamma(t) \int_{0}^{t} \exp \left(\frac{b}{\beta \kappa} \int_{s}^{t} \Gamma(\tau) d \tau\right) d s\right] .
\end{aligned}
$$

Integration of this inequality with the use of (36) implies that there exists a continuous, monotonically increasing, positive function $M(t)$ such that for any $t \geq 0$

$$
L_{6}(t) \leq M(t)\left[\left(a_{0}+\beta \bar{a}(0)\right) u^{2}(0)+(1+\beta) \dot{u}^{2}(0)\right] .
$$

Since $\lim _{t \rightarrow \infty} Q(t)=Q(\infty), \lim _{t \rightarrow \infty} \dot{Q}(t)=0$ and the functions $F_{1}(t), F_{2}(t)$ are bounded, for any $\epsilon>0$ there exists $t_{0}(\epsilon)>0$ such that for any $t \geq t_{0}(\epsilon)$ :

$$
\left|G(t)-G_{\infty}(t)\right|<\epsilon,
$$

where

$$
G_{\infty}(t)=\beta\left[H(\infty)-\frac{\dot{a}(t)}{\bar{a}(t) b}\right]+3 F_{1}(t) Q(\infty)
$$

Estimating this function we find

$$
G_{\infty}(t) \geq \beta\left[H(\infty)-Y-3 \beta^{-1}|Q(\infty)| Y_{1}\right] .
$$

From this inequality, (17), and (18) it follows that there exists an $\epsilon_{1}>0$ such that $G_{\infty}(t) \geq 2 \epsilon_{1}$ for any $t \geq 0$. Choosing $\epsilon=\epsilon_{1}$ and applying (39) we obtain that $G(t) \geq \epsilon_{1}$ for $t \geq t_{0}\left(\epsilon_{1}\right)$. This inequality and (33) imply $\dot{L}_{6}(t) \leq-\kappa \epsilon_{1} b u^{2}(t)$ for $t \geq t_{0}\left(\epsilon_{1}\right)$. From this relationship and (35) it follows that

$$
\kappa\left[\beta u^{2}(t)+\epsilon_{1} b \int_{t_{0}\left(\epsilon_{1}\right)}^{t} u^{2}(s) d s\right] \leq L_{6}\left(t_{0}\left(\epsilon_{1}\right)\right), \quad t \geq t_{0}\left(\epsilon_{1}\right) .
$$

Estimating $L_{6}\left(t_{0}\left(\epsilon_{1}\right)\right)$ with the use of (38) we finally find that for any $t \geq t_{0}\left(\epsilon_{1}\right)$ :

$$
\begin{aligned}
& \kappa\left[\beta u^{2}(t)+\epsilon_{1} b \int_{t_{0}\left(\epsilon_{1}\right)}^{t} u^{2}(s) d s\right] \\
& \quad \leq M\left(t_{0}\left(\epsilon_{1}\right)\right)\left[\left(a_{0}+\beta \bar{a}(0)\right) u^{2}(0)+(1+\beta) \dot{u}^{2}(0)\right] .
\end{aligned}
$$

This inequality implies the theorem.

REMARK. Let us consider the exponential kernel of the integral operator

$$
Q(t)=-\chi[1-\exp (-\gamma t)]
$$

where $\chi$ and $\gamma$ are constant, $0<\chi<1, \gamma>0$. In this case, the conditions (i), (ii) are true, and the inequalities (16)-(18) can be represented as follows:

$$
\min _{t \geq 0} a(t)>\chi b, \quad Y_{1} \leq \frac{\gamma}{6} Y_{2}, \quad Y<\frac{\gamma Y_{2}-6 Y_{1}}{\gamma Y_{2}-3 Y_{1}} \frac{\chi}{\gamma} .
$$


4. Connection between stability of the integro-differential equation (1) and the corresponding ordinary differential equation (5). The above stability conditions for Eq. (1) are formulated in such a way that, at first sight, they have no connection with the well-known stability condition (13) for Eq. (5). In this section it is demonstrated that our conditions of asymptotic stability for Eq. (1) are fulfilled if and only if the zero solution of (5) is stable and the functions $a(t)$ and $Q(t)$ satisfy some additional conditions. We restrict ourselves to the consideration of the noncritical case when all eigenvalues of the matrix $\Phi(T)$ are single.

Denote by $X(t)$ the matrix function with the components $X_{i j}(t)$, and by $\phi(n)$ the matrix function with the components $\phi_{i j}(n)$. The function $X(t)$ is determined in $[0, T]$ and coincides with $\Phi(t)$ in this interval. The function $\phi(n)$ is determined for a nonnegative integer $n$ and equals $\Phi(n T)$. It can be shown that

$$
\Phi(t)=X(t-n T) \phi(n), \quad n T \leq t<(n+1) T .
$$

Putting $t=(n+1) T$ we obtain from $(43)$ that $\phi(n+1)=\Phi(T) \phi(n)$. Since $\phi(0)=$ $\Phi(0)=I$, this equality implies that

$$
\phi(n)=\Phi^{n}(T)
$$

Substitution of (42) into (14) yields

$$
\begin{aligned}
& f_{1}(t)=a_{0}\left[X_{11}(t-n T) X_{12}(t-n T) \phi_{11}^{2}(n)\right. \\
& +\left(X_{11}(t-n T) X_{22}(t-n T)+X_{12}(t-n T) X_{21}(t-n T)\right) \phi_{11}(n) \phi_{12}(n) \\
& \left.+X_{21}(t-n T) X_{22}(t-n T) \phi_{12}^{2}(n)\right] \\
& +\left[X_{11}(t-n T) X_{12}(t-n T) \phi_{21}^{2}(n)\right. \\
& +\left(X_{11}(t-n T) X_{22}(t-n T)+X_{12}(t-n T) X_{21}(t-n T)\right) \phi_{21}(n) \phi_{22}(n) \\
& \left.+X_{21}(t-n T) X_{22}(t-n T) \phi_{22}^{2}(n)\right] \text {. }
\end{aligned}
$$

Let us consider the quadratic form

$$
l(t, \xi, \eta)=b_{11}(t) \xi^{2}+2 b_{12}(t) \xi \eta+b_{22}(t) \eta^{2}
$$

where

$$
\begin{aligned}
& b_{11}(t)=X_{11}(t-n T) X_{12}(t-n T) \\
& b_{12}(t)=X_{11}(t-n T) X_{22}(t-n T)+X_{12}(t-n T) X_{21}(t-n T), \\
& b_{22}(t)=X_{21}(t-n T) X_{22}(t-n T)
\end{aligned}
$$

From $(6)$ and $(11)$ it follows that $\operatorname{det} X(t)=\operatorname{det} X(0)=1$ for any $t \in[0, T]$. From (8) it follows that there exists a sufficiently small $t_{1}>0$ such that $X_{11}\left(t_{1}\right)>0, X_{12}\left(t_{1}\right)<0$. Therefore, for $t=t_{1}+n T$, the form $l(t, \xi, \eta)$ is negatively defined, and there is a $\delta>0$ such that $l\left(t_{1}+n T, \xi, \eta\right) \leq-\delta\left(\xi^{2}+\eta^{2}\right)$. This means that

$$
f_{1}\left(t_{1}+n T\right) \leq-\delta\left[a_{0}\left(\phi_{11}^{2}(n)+\phi_{12}^{2}(n)\right)+\left(\phi_{21}^{2}(n)+\phi_{22}^{2}(n)\right)\right] .
$$


From this inequality it follows that there exists a $\delta_{1}>0$ such that

$$
\left|f_{1}\left(t_{1}+n T\right)\right| \geq \delta_{1}\|\phi(n)\|^{2}
$$

Inequalities (43) and (44) imply

$$
\sup _{t \geq 0}\left|f_{1}(t)\right| \geq \delta_{1} \max _{n}\left\|\Phi^{n}(T)\right\|^{2}
$$

For any positive integer $n$, we have $\left\|\Phi^{n}(T)\right\| \geq \rho^{n}(\Phi(T))$, where $\rho$ is the spectral radius of the matrix; cf., e.g., Horn and Johnson (1985). This inequality together with (45) leads to the estimate

$$
\sup _{t \geq 0}\left|f_{1}(t)\right| \geq \delta_{1} \max _{n} \rho^{2 n}(\Phi(T))=\delta_{1} \max _{n} \rho^{2 n}(\Psi(T))
$$

From (14) it follows that

$$
\inf _{t \geq 0} f_{2}(t) \leq f_{2}(0)=1 \text {. }
$$

According to (46) and (47), the stability condition (17) holds only for $\rho(\Psi(T)) \leq 1$. On the other hand, it is known, see, e.g., Yakubovich and Starzhinskii (1975), that $\rho(\Psi(T)) \geq 1$. Therefore, the inequality (17) implies $\rho(\Psi(T))=1$. For the noncritical case, the necessary and sufficient stability condition (13) for Eq. (5) follows from this equality and (12). Hence, we have proved that for the noncritical case, the stability conditions for the integro-differential equation (1) imply the stability conditions for the corresponding differential equation (5).

Now we derive an inverse result; namely, we prove that the stability of the ordinary differential equation (5) ensures the stability of the integro-differential equation (1) under some assumptions about the behavior of the kernel $Q(t)$ of the integral operator and the periodic coefficient $a(t)$.

The stability of the equation (5) implies the boundedness of the fundamental matrix function $\Phi(t)$. This means that there exists a positive constant $c_{1}$ such that for any $t \geq 0$ :

$$
\left|\Phi_{i j}(t)\right| \leq c_{1} \quad(i, j=1,2)
$$

It follows from this inequality and (14) that there exists a positive constant $c_{2}$ such that for any $t \geq 0$ :

$$
\left|f_{1}(t)\right| \leq c_{2}, \quad 0 \leq f_{2}(t) \leq c_{2}, \quad 0 \leq f_{3}(t) \leq c_{2} .
$$

Let us prove that there exists a positive constant $c_{3}$ such that for any $t \geq 0$ :

$$
f_{2}(t)>c_{3}
$$

Suppose that this hypothesis is not true. Therefore, there exists a sequence $\left\{t_{m}\right\}$ such that $f_{2}\left(t_{m}\right)<m^{-2}$. From this inequality and (14) it follows that

$$
\left|\Phi_{12}\left(t_{m}\right)\right| \leq c_{4} m^{-1}, \quad\left|\Phi_{22}\left(t_{m}\right)\right| \leq c_{4} m^{-1},
$$


where $c_{4}=\max \left(1, a_{0}^{-1 / 2}\right)$. These estimates and the condition (11):

$$
\operatorname{det} \Phi\left(t_{m}\right)=\Phi_{11}\left(t_{m}\right) \Phi_{22}\left(t_{m}\right)-\Phi_{12}\left(t_{m}\right) \Phi_{21}\left(t_{m}\right)=1
$$

imply

$$
\begin{aligned}
1 & \leq c_{4} m^{-1}\left[\left|\Phi_{11}\left(t_{m}\right)\right|+\left|\Phi_{12}\left(t_{m}\right)\right|\right] \\
& \leq 2 c_{4} m^{-1} \sqrt{\Phi_{11}^{2}\left(t_{m}\right)+\Phi_{12}^{2}\left(t_{m}\right)} \leq 2 c_{4}^{2} m^{-1} \sqrt{f_{3}\left(t_{m}\right)}
\end{aligned}
$$

Therefore, $f_{3}\left(t_{m}\right) \geq\left[m /\left(2 c_{4}^{2}\right)\right]^{2}$. Since this inequality contradicts (48), our assumption is not true and the inequality (49) holds.

It follows from the formulae (48), (49) that the stability condition (13) for the ordinary differential equation (5) yields $Y_{1} \leq c_{2}, Y_{2} \geq c_{3}$. In this case, the inequality (17) can be treated only as a restriction on the function $Q(t)$ which guarantees asymptotic stability of the zero solution of (1). For a given kernel $Q(t)$ satisfying (17), the inequality (18) can be considered as a restriction on the behavior of the coefficient $a(t)$ which ensures asymptotic stability.

5. Stability of a viscoelastic beam under periodic compressive load. In this section we derive stability conditions for a rectilinear viscoelastic beam under the action of a compressive load.

Let us consider the plane bending of a viscoelastic beam with the length $l$, the crosssectional area $S$, and the moment of inertia of the cross section $J$. At the moment $t=0$, the compressive forces $P=P(t)$ are applied to the beam ends. Under the action of the external forces, the beam deforms. Denote by $y(t, x)$ the beam deflection at the point with the longitudinal coordinate $x$ at the moment $t \geq 0$. We suppose that (a) the function $y$ and its derivative are sufficiently small, and we can neglect the nonlinear terms in the expression for the curvature of the longitudinal axis; (b) the hypothesis regarding plane sections in the bending is fulfilled.

We assume that for a uniaxial stress state in the beam, the stress $\sigma(t)$ is connected with the strain $e(s)(0 \leq s \leq t)$ by the constitutive equation of a linear viscoelastic solid, cf. Christensen (1982):

$$
\sigma(t)=E\left[e(t)+\int_{0}^{t} \dot{Q}(t-s) e(s) d s\right]
$$

where $E$ is the constant Young modulus and $Q(t)$ is the relaxation measure satisfying the conditions (i) and (ii); see Sec. 2. Some examples of the relaxation measures of polymers, plastics, concrete, and soils are represented in Arutyunyan et al. (1987), Christensen (1982).

It has been shown, see, e.g., Drozdov et al. (1991), that the function $y(t, x)$ satisfies the equation

$$
\rho S \ddot{y}(t, x)+E J\left[D^{4} y(t, x)+\int_{0}^{t} \dot{Q}(t-s) D^{4} y(s, x) d s\right]+P(t) D^{2} y(t, x)=0
$$


with the initial conditions $y(0, x)=v_{1}(x), \dot{y}(0, x)=v_{2}(x)$. Here $\rho$ is the mass density, $v_{1}(x)$ is the initial deflection of the beam, $v_{2}(x)$ is the initial speed of deflection, $D$ is the operator of differentiation with respect to $x, D y=\partial y / \partial x$.

We restrict ourselves to the consideration of the simply-supported beam, when the boundary conditions can be written as follows:

$$
y(t, 0)=y(t, l)=0, \quad D^{2} y(t, 0)=D^{2} y(t, l)=0
$$

In order to satisfy these conditions we seek the solution of Eq. (50) in the form

$$
y(t, x)=\sum_{n=1}^{\infty} u_{n}(t) \sin \frac{\pi n x}{l}
$$

Substituting (51) into (50) we obtain

$$
\rho S \ddot{u}_{n}(t)+\frac{\pi^{4} n^{4} E J}{l^{4}}\left[\left(1-\frac{P(t)}{P_{e} n^{2}}\right) u_{n}(t)+\int_{0}^{t} \dot{Q}(t-s) u_{n}(s) d s\right]=0,
$$

where $P_{e}=\pi^{2} E J l^{-2}$ is the Euler critical force.

Suppose that the load $P(t)$ has the form $P(t)=P_{0}+P_{1} \sin (\Omega t)$, where $P_{0}, P_{1}$, and $\Omega$ are positive constants. In the following, the main stability region is analysed. To this end, only the term with $n=1$ is considered, and Eq. (52) implies

$$
\ddot{u}\left(t_{*}\right)+\left[1+\mu \sin \left(\omega t_{*}\right)\right] u\left(t_{*}\right)+\int_{0}^{t_{*}} \dot{Q}_{*}\left(t_{*}-s_{*}\right) u\left(s_{*}\right) d s_{*}=0,
$$

where

$$
t_{*}=t / \mathcal{T}, \quad \omega=\Omega \mathcal{T}, \quad \mathcal{T}=\sqrt{\frac{\rho S l^{4}}{\pi^{4} E J}}, \quad \mu=\frac{P_{1}}{P_{e}-P_{0}}, \quad Q_{*}\left(t_{*}\right)=\frac{P_{e} Q(t)}{P_{e}-P_{0}} .
$$

Let us restrict ourselves to the stability analysis for a beam made of the standard viscoelastic material with the relaxation measure (40). Applying the Theorem to Eq. (53) we obtain the following stability conditions:

$$
1-\mu>P_{e} \chi\left(P_{e}-P_{0}\right)^{-1}, \quad \gamma_{1}<\gamma<\gamma_{2}
$$

where $\gamma_{1}$ and $\gamma_{2}$ are the roots of the quadratic equation

$$
Y Y_{2} \gamma^{2}-\left(3 Y Y_{1}+\chi Y_{2}\right) \gamma+6 \chi Y_{1}=0
$$

The boundaries of the stability region in the $(\chi, \gamma)$-plane are plotted in Fig. 1 . The calculations are carried out for $P_{0}=0.2 P_{e}$ and $P_{1}=0.1 P_{e}$ (that corresponds to $\mu=$ 0.125 ) and for $\omega=0.2$ (black points), $\omega=0.5$ (light points), and $\omega=0.8$ (asterisks). The numerical analysis shows that the lower boundary of the stability region is very close to the abscissa axis practically for the whole range of $\omega$ values. The upper boundary of this region has essential maximum for $\chi \approx 0.35$ that corresponds to half the maximal 
10

5

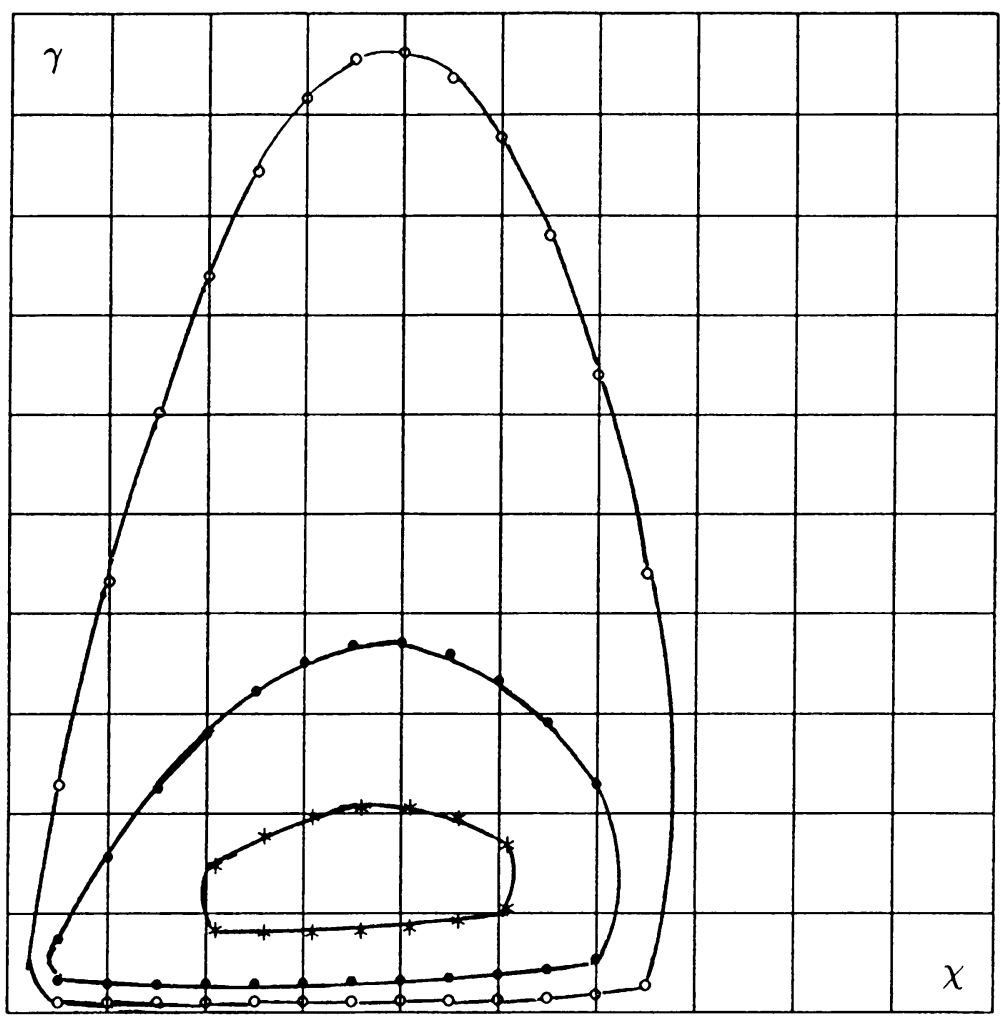

0

0.5

FIG. 1. Stability domain for a beam driven by periodic excitations. Light points- $\omega=0.2$, black points- $-\omega=0.5$, asterisks- $-\omega=0.8$.

admissible $\chi$ value. With the growth of the frequency $\omega$, the stability region significantly decreases and disappears for $\omega>1$. This means that for a fixed intensity of compressive load a beam driven by periodic forces is stable only for sufficiently small frequencies of periodic excitations.

6. Conclusions. We consider the stability problem for a linear integro-differential equation (1) with periodic coefficients. Equation (1) arises in the dynamics of viscoelastic thin-walled elements of structures. This equation has two specific properties: (a) in the absence of the integral term it has no reserve of stability, (b) the kernel of the integral term satisfies some restrictions following from the second law of thermodynamics, which ensure the asymptotic stability of Eq. (1).

Using the direct Lyapunov method and constructing the stability functionals we derive some sufficient stability conditions for Eq. (1). When the integral term vanishes, these conditions coincide with the stability criterion for the ordinary differential equation into which Eq. (1) turns. In the general case, the stability conditions that were suggested mean that a viscoelastic structure is asymptotically stable under periodic loads if the 
corresponding elastic structure is stable and the rate of the stress relaxation is sufficiently large.

As an example, we have considered the stability problem for a rectilinear viscoelastic beam under the action of periodic compressive forces applied to the beam ends, and we have formulated a limitation on the relaxation rate that ensures the asymptotic stability of the beam. We have studied numerically the dependence of the critical relaxation rate on the frequency of the periodic load.

\section{REFERENCES}

[1] N. Kh. Arutyunyan, A. D. Drozdov, and V. E. Naumov, Mechanics of Growing Viscoelastoplastic Bodies, Nauka, Moscow, 1987 (in Russian)

[2] L. Kh. Belen'kaya and V. I. Judovich, Stability of a viscoelastic rod under a periodic load, Mech. Solids 13, no. 6, 128-133 (1978)

[3] F. Bloom, On the existence of solutions to nonstrictly hyperbolic problems in nonlinear viscoelasticity, Applicable Analysis 17, 115-133 (1984)

[4] V. V. Bolotin, Dynamic Stability of Elastic Systems, Holden Day, San Francisco, 1964

[5] T. A. Burton, Volterra Integral and Differential Equations, Academic Press, New York, 1983

[6] G. Cederbaum and M. Mond, Stability properties of a viscoelastic column under a periodic force, Trans. Amer. Soc. Mech. Eng. J. Appl. Mech. 59, 16-19 (1992)

[7] B. D. Coleman and D. Hill, On the stability of certain motions of incompressible materials with memory, Arch. Rational Mech. Anal. 30, no. 3, 197-224 (1968)

[8] C. Corduneanu and V. Lakshmikantham, Equations with infinite delay. A survey, Nonlinear Anal. 4, 831-877 (1980)

[9] R. M. Christensen, Theory of Viscoelasticity. An Introduction, Academic Press, New York, 1982

[10] C. M. Dafermos, An abstract Volterra equation with applications to linear viscoelasticity, J. Differential Equations 7, 554-569 (1970)

[11] C. M. Dafermos and J. A. Nohel, Energy methods for nonlinear hyperbolic Volterra integrodifferential equations, Comm. Partial Differential Equations 4, 219-278 (1979)

[12] A. Drozdov, Stability of viscoelastic shells under periodic and stochastic loading, Mech. Res. Comm. 20, no. 6, 481-486 (1993)

[13] A. D. Drozdov and V. B. Kolmanovskii, Stochastic stability of viscoelastic bars, Stochastic Anal. Appl. 10, no. 3, 265-276 (1992)

[14] A. D. Drozdov, V. B. Kolmanovskii, and P. A. Velmisov, Stability of Viscoelastic Systems, Saratov Univ. Press, Saratov, 1991 (in Russian)

[15] N. P. Erugin, Linear Systems of Ordinary Differential Equations with Periodic and Quasi-Periodic Coefficients, Academic Press, New York, 1966

[16] Kh. Eshmatov and P. Kurbanov, Parametric oscillations in a viscoelastic rod with a nonlinear inheritance characteristic, Appl. Math. Mech. 39, no. 4 (1975)

[17] R. M. Evan-Iwanowski, Resonance Oscillations in Mechanical Systems, Elsevier, New York, 1976

[18] M. Fabrizio and B. Lazzari, On the existence and the asymptotic stability of solutions for linearly viscoelastic solids, Arch. Rational Mech. Anal. 116, no. 2, 139-152 (1991)

[19] M. Fabrizio and A. Morro, Mathematical Problems in Linear Viscoelasticity, SIAM Studies in Applied Mathematics 12 (1992)

[20] G. Gripenberg, S. O. Londen, and O. Staffans, Volterra Integral and Functional Equations, Cambridge Univ. Press, Cambridge, 1990

[21] J. K. Hale, Theory of Functional Differential Equations, Springer-Verlag, New York, 1977

[22] G. Herrmann, Dynamic Stability of Structures, Pergamon Press, Oxford, 1967

[23] R. A. Horn and C. R. Johnson, Matrix Analysis, Cambridge Univ. Press, Cambridge, 1985

[24] V. B. Kolmanovskii and V .R. Nosov, Stability of Functional Differential Equations, Academic Press, New York, 1986

[25] R. C. MacCamy, A model for one-dimensional nonlinear viscoelasticity, Quart. Appl. Math. 35, no. 1, 1-19 (1977)

[26] I. G. Malkin, Some Problems of the Theory of Nonlinear Oscillations, Gostechizdat, Moscow, 1956 (in Russian) 
[27] V. I. Matyash, Dynamic stability of a hinged viscoelastic bar, Mech. Polymers 2, 293-300 (1967)

[28] N. W. Mclachlan, Theory and Applications of Mathieu Functions, Dover Publications, New York, 1964

[29] M. Renardy, W. J. Hrusa, and J. A. Nohel, Mathematical Problems in Viscoelasticity, Longmans Press, Essex, 1987

[30] K. K. Stevens, On the parametric excitation of a viscoelastic column, AIAA J. 12, 2111-2116 (1966)

[31] A. Stokes, A Floquet theory for functional differential equations, Proc. Nat. Acad. Sci. USA 48, 1330-1334 (1962)

[32] V. A. Yakubovich and V. M. Starzhinskii, Linear Differential Equations with Periodic Coefficients, Vols. 1, 2, John Wiley, New York, 1975 